\title{
Transient radio emission from PSR B1259-63
}

\author{
Lewis Ball ${ }^{1}$, Andrew Melatos ${ }^{2}$, Simon Johnston ${ }^{1}$, Olaf Skjæraasen ${ }^{1,3}$ \\ ${ }^{1}$ Research Centre for Theoretical Astrophysics, Univ. Sydney, Australia \\ ${ }^{2}$ Miller Fellow, Dept. of Astronomy, Univ. California, Berkeley, USA \\ ${ }^{3}$ Institute of Theoretical Astrophysics, Univ. Oslo, Norway
}

\begin{abstract}
The unique binary pulsar system PSR B1259-63, comprising a radio pulsar in an eccentric orbit about a hot, massive companion star, has been extensively monitored at radio frequencies between $843 \mathrm{MHz}$ and $8.4 \mathrm{GHz}$ during its 1994 and 1997 periastron passages. Unpulsed radio emission was detected for about 100 days around each periastron, varying on time scales as short as a day. We argue that the emission is synchrotron radiation generated as the pulsar passes through the Be-star's excretion disk just before and just after periastron.
\end{abstract}

\section{Introduction}

The binary pulsar PSR B1259-63 is in a highly eccentric $(e \sim 0.87), 3.5-\mathrm{yr}$ orbit around a 10th magnitude B2e star, SS 2883, of radius $R_{*} \sim 6 R_{\odot}$. This is the only radio pulsar orbiting a main-sequence companion with a circumstellar disk. At periastron the pulsar's orbital radius is $23 R_{*}$. The disk extends to at least $20 R_{*}$ (Johnston et al. 1994), and is steeply inclined to the orbit plane (Wex et al. 1998). Melatos, Johnston \& Melrose (1995) proposed a wind-disk model of the system to explain orbital variations of the flux density, polarisation, rotation measure and dispersion measure of the pulsed emission near periastron (Johnston et al. 1996). In the model, the eclipse of the pulsed emission between $\mathcal{T}-20$ and $\mathcal{T}+16$ (where $\mathcal{T}$ denotes the epoch of periastron) is attributed to free-free absorption in the disk.

\section{Radio observations}

Unpulsed radio emission was detected from PSR B1259-63 around the 1994 and 1997 periastron passages (Johnston et al. 1999). In 1997 the emission appeared suddenly at $\mathcal{T}-22$, the flux density $S$ having previously been below $0.5 \mathrm{mJy}(3 \sigma)$ at all frequencies. There followed a complicated evolution peaking at $S \sim 60 \mathrm{mJy}$ at $1.4 \mathrm{GHz}$ around $\mathcal{T}+18$ - see Fig. 1 . The 1994 data are similar and showed detectable emission until $\mathcal{T}+100$.

At most times the radio frequency spectrum is well fitted by a power law $S \propto \nu^{-\alpha}$ of spectral index $\alpha \approx 0.5$, consistent with synchrotron radiation from relativistic electrons. There is evidence in both the 1994 and 1997 data that the 

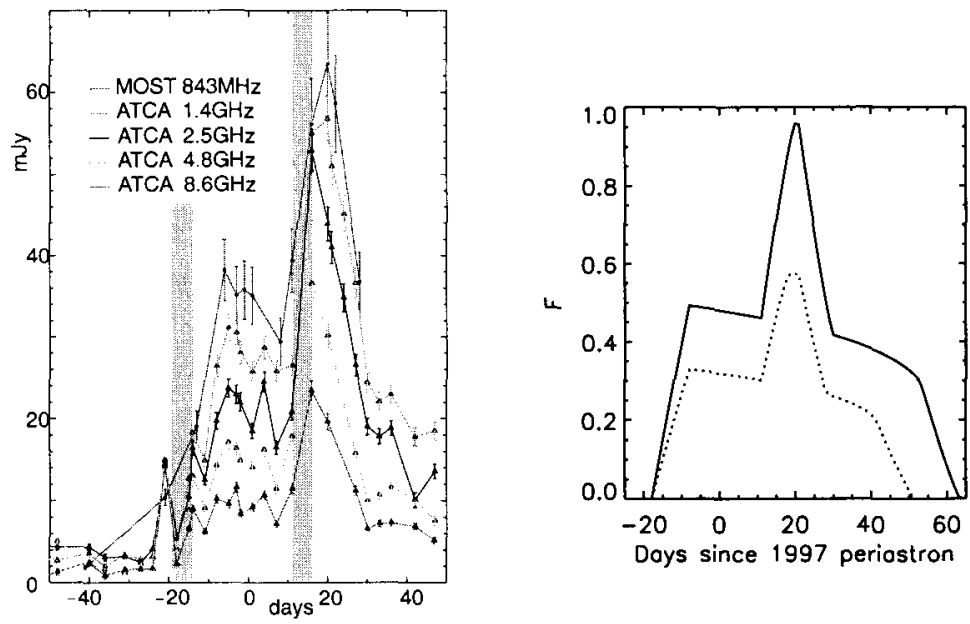

Figure 1. Left: Unpulsed radio emission from PSR B1259-63 around the 1997 periastron. Right: Model light curves.

spectrum steepens after periastron. There is no compelling evidence for a low frequency turnover in the spectrum at any epoch between $\mathcal{T}-22$ and $\mathcal{T}+47$.

\section{Interpretation}

The detection of unpulsed radio emission through periastron, when the pulsed emission is eclipsed and the pulsar is believed to be obscured behind the dense Be-star disk, suggests that the source of the emission does not closely track the motion of the pulsar. Estimates of the magnetic field strengths due to the pulsar and Be-star fields allow estimates of the required Lorentz factors and number density of the radiating electrons. These are consistent with the emission originating from electrons accelerated from the Be-star outflow, but not with the likely parameters of electrons from the pulsar wind.

A model of radio emission from electrons accelerated from the disk during the two pulsar-disk crossings, and subject to significant synchrotron losses, is consistent with the present understanding of the geometry of the system and provides a good qualitative match to the observations (Ball et al. 1999).

\section{References}

Ball, L., Melatos, A., Johnston, S., \& Skjæraasen, O., 1999, ApJ, 514, L39

Johnston, S., et al., 1994, MNRAS, 268, 430

Johnston, S., et al., 1996, MNRAS, 279, 1026

Johnston, S., et al., 1999, MNRAS, 302, 277

Melatos, A., Johnston, S., \& Melrose, D. B., 1995, MNRAS, 275, 381

Wex, N., et al.., 1998, MNRAS, 298, 997 